\title{
A Magneto-optical Trap Below a Dielectric Coated Mirror Surface
}

\author{
Hoon Yu, Lim Lee, Kyung Hyun Lee, and Jung Bog Kim* \\ Department of Physics Education, Korea National University of Education, \\ Chung-Buk 363-791, Korea
}

(Received February 5, 2008 : revised April 30, 2008 : accepted April 30, 2008)

\begin{abstract}
A Magneto-Optical Trap (MOT) for ${ }^{87} \mathrm{Rb}$ atoms near the surface of a dielectric coated mirror at the top of a small $20 \times 25 \times 40 \mathrm{~mm}^{3}$ cell has been observed. Two beams of $3.3 \mathrm{~mW} / \mathrm{cm}^{2}$ were used for optical cooling and an anti-Helmholtz magnetic field with a spatial gradient of $9.1 \mathrm{G} / \mathrm{cm}$ was used for magnetic trapping. The thickness of the mirror coated on a cover glass was less than $100 \mu \mathrm{m}$. The mirror covered the top of a cell and the atom-chip was located outside the vacuum in order to exploit the long life time of the mirror and easy operation of the chip. The trapping position was found $5 \mathrm{~mm}$ beneath the mirror surface. The number of trapped atoms was roughly $3 \times 10^{7}$ atoms and the temperature was approximately a few tens mK. In this paper, we describe the construction of the mirror-MOT in detail.
\end{abstract}

Keywords: Rb, Magneto-optical trap (MOT), Mirror magneto-optical trap (M-MOT)

OCIS codes : (020.0020) Atomic and molecular physics; (020.7010) Trapping

\section{INTRODUCTION}

Albert Einstein proposed two wave coherence theories for a massless photon and massive bosonic atoms in $1916[1,2]$. The first microwave laser, called MASER, was later realized in 1954. Numerous studies of stimulated emission have led to major advances in laser technology, which have in turn found applications related to our daily life. In 1987, a major step toward realization of Bose-Einstein Condensation was taken when a Magneto-Optical Trap of neutral sodium was achieved by Pritchard [3]. Ketterle, Cornell, and Wieman subsequently observed Bose-Einstein Condensation (BEC) from trapped atoms in $1995[4,5]$.

The normal method for BEC uses atoms transferred from a traditional six beam MOT to a magnetic trap. However, the geometrical size of the system could not be reduced, and some atoms are lost during the transfer. Furthermore, since the system requires many optical components, improvements were needed to allow practical applications such as a laser. Many researchers have thus focused on developing a smaller and simpler system. While research to miniaturize the condensation system

*Corresponding author: jbkim@knue.ac.kr using an atom chip was reported in 2001, this approach was limited by the atom chip being installed inside the vacuum chamber [6,7]. Installation of the atom chip outside the vacuum chamber would lend many advantages, such as a much more miniaturized and even portable system. In 2005, condensation of $\mathrm{Rb}$ atoms was realized from an external atom chip [8]. Some disadvantage remained, however, primarily relating to the use of a metal mirror on the external atom chip. Specifically, the lifetime of the metal mirror was short and maintenance of polarization was poor as compared to the use of a dielectric coated mirror.

In the present research, we were able to produce a Mirror-Magneto-Optical Trap (M-MOT) with a dielectric coated mirror, which offers longer lifetime and better polarization maintenance than the metal mirror. M-MOT is the first step in developing an external atom chip, since it offers the advantage of placing the MOT near the mirror surface, where the circuit for the magnetic trap is located. Furthermore, baking time could be reduced to just one week for a $10^{-9}$ torr vacuum and operating time was similarly shortened by using only an $\mathrm{Rb}$ getter instead of a dispenser. Thus, the present MMOT system for BEC applications provides notable improvements over the conventional approaches. In this 
paper, we describe construction of the M-MOT in detail.

\section{EXPERIMENTAL SETUP}

\section{Vacuum chamber}

Fig. 1 (a) shows the configuration of the mirror, the atom chip, and the cuvette in terms of attachment to the vacuum chamber for the M-MOT. An electric circuit under the atom chip was printed for the Z-type magnetic trap. The atom chip and the cuvette are separated by a mirror for the following reasons: (i) to reduce the disturbance of trapped atoms due to the atom chip's current heat; (ii) to prevent degradation of the vacuum due to outgassing from the atom chip; and (iii) to readily supply electric power for the magnetic trap. Therefore, the mirror must be stable under a high vacuum of at least $10^{-9}$ torr and be thin enough to create an efficient magnetic field gradient for the magnetic trap by the atom chip. The dielectric coated mirror shows longer endurance under a high vacuum and can better maintain high polarization for reflection than the metal mirror [9]. Given these conditions, a dielectric coated cover glass of $100 \mu \mathrm{m}$ thickness is used.

The cuvette of $20 \times 25 \times 40 \mathrm{~mm}^{3}$ was made of Pyrex glass. The cuvette was manufactured by bonding four Pyrex plates by using Epo-Tek 353ND instead of by melting adhesion, because heat can render the cuvette's surface too inhomogeneous, which is not appropriate for maintaining polarization of light for a MOT. The lower part of the cuvette is connected to a glass plate, which has a circular hole in its center. The plate is again connected to the Pyrex tube, which is sealed with a $2.75^{\prime \prime}$ stainless steel flange (MDC comp.) The vacuum chamber consists of a $2.75^{\prime \prime} 6$-way cross, a gate valve, an ion gauge, a feed through flange for current supply to an Rb getter, an ion pump, and a flange for the M-MOT. The gate valve is connected to a rotary pump through a turbo molecular pump for rough pumping. Fig. 1 (b) shows the vacuum chamber. For baking, first, a 6 -way cross was heated up to $300^{\circ} \mathrm{C}$. The turbo and the ion pumps were then heated to $250^{\circ} \mathrm{C}$. The cuvette was heated up to $150^{\circ} \mathrm{C}$ during 24 hours. Each temperature was thereafter maintained for $3 \sim 4$ days. Finally, a $5 \sim 9 \times 10^{-9}$ torr, which is suitable for M-MOT,

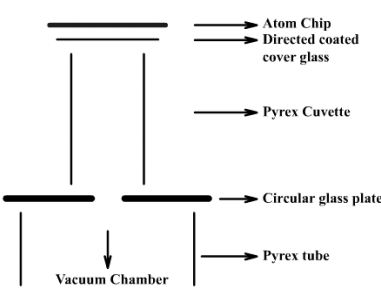

(a)

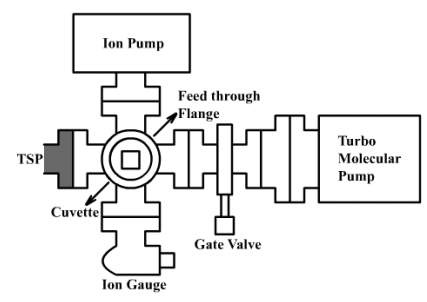

(b)
FIG. 1. (a) Structure of a cuvette for the mirror MOT, (b) vacuum chamber. was reached.

\section{Laser setup for M-MOT}

We used three diode lasers - cooling, repumping, and probing beams - as shown in Fig. 2. The cooling beam was obtained from a MOPA (TOPICA Model;TA500) system for high power, i.e., $450 \mathrm{~mW}$. After the cooling beam was modulated twice by an $\mathrm{AOM}$ of $60 \mathrm{MHz}$, it was coupled to the optical fiber. Finally, we could obtain of $13 \mathrm{~mW}$ cooling beam for the M-MOT. Low power diode lasers (DL100, TOPICA) were used for repumping and probing. Each laser supplied sufficient power, i.e., $30 \mathrm{~mW}$ at $780 \mathrm{~nm}$, after passing through an optical isolator. AOM 1 and 2 in Fig. 2 were used to change each laser's frequency for frequency detuning and create a laser pulse in order to measure the number of trapped atoms.

Frequencies of the lasers were evaluated on the basis of the saturated absorption spectrum using a $\mathrm{Rb}$ vapor cell and were stabilized by the lock-in-stabilization method. The cooling laser's frequency was locked to the crossover line of ${ }^{87} \mathrm{Rb}$ between the $|\mathrm{F}=2>\rightarrow| \mathrm{F}^{\prime}=3>$ and $\mid \mathrm{F}=2>$ $\rightarrow \mid F^{\prime}=2>$ transition. The red detuning of the cooling beam for the MOT was adjusted by double pass of a $60 \mathrm{MHz} \mathrm{AOM}$. The laser frequency was red-detuned by $7.1 \mathrm{MHz}$ to the $|\mathrm{F}=2>\rightarrow| \mathrm{F}^{\prime}=3>$ cooling transitions, which is almost ideal detuning for a MOT. The probe laser was locked to the same transition as the cooling laser; however, it passes AOM2 $(+70 \mathrm{MHz})$ and AOM3 $(+80 \mathrm{MHz})$ so as to be resonant with the $\mid \mathrm{F}=2>\rightarrow$ $\mid F^{\prime}=3>$ transition. The repumping laser was locked to the $|\mathrm{F}=1>\rightarrow| \mathrm{F}^{\prime}=2>$ transition. These cooling and repumping beams were combined with a polarization beam splitter cube, and then coupled into a single mode optical fiber to be sent to the M- MOT. Fig. 3 shows

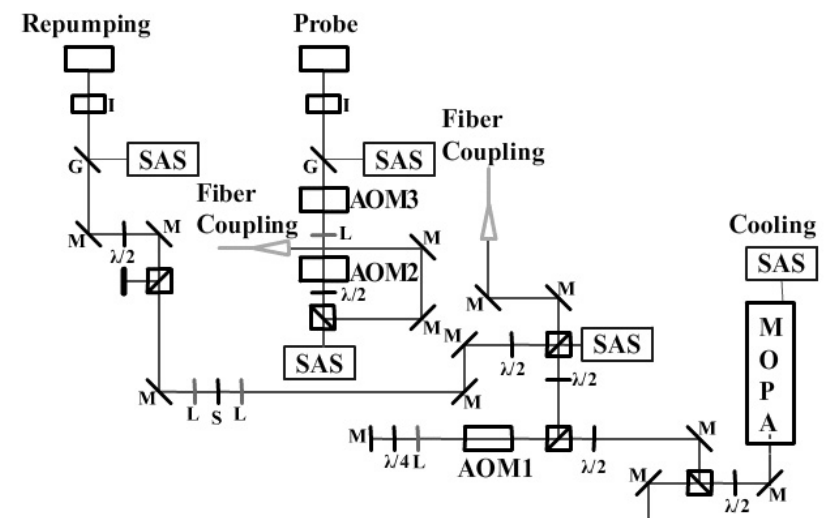

FIG. 2. Laser alignments for M-MOT. AOM1 $(+60 \mathrm{MHz})$ is used for the detuning frequency of the cooling laser and shuttering. The cooling laser overlapped with the repumping beam is then coupled into an optical fiber. The probe beam is controlled by AOM2 $(+70 \mathrm{MHz})$ and AOM3 $(+60 \mathrm{MHz})$. 


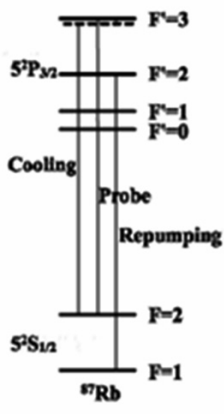

(a)

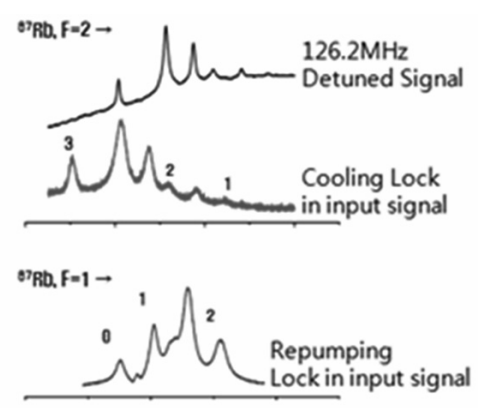

(b)
FIG. 3. (a) Each laser frequency selection for M-MOT (b) ${ }^{87} \mathrm{Rb}$ SAS signals.

the ${ }^{87} \mathrm{Rb}$ saturated absorption spectrum (SAS) lines obtained using a vapor cell.

\section{Optical alignment}

Fig. 4 (a) shows an M-MOT point where the trapping beams overlap. We send only two pairs of beams into the cuvette to create the M-MOT. In Fig. 4(a), one input beam traveling upward toward the mirror is reflected by the dielectric coated internal mirror. The downward beam is retro-reflected by the external mirror. Reflected beams by the internal mirror act as two more beams in the conventional MOT, as shown in Fig. 4(b). One input beam is sufficient to act as four beams. Two more beams, for a total of six beams, are aligned in the same manner as a six beam MOT. These two additional beams are shown in Fig. 4(a), and their directions are vertical to the sheet. No difference between the conventional MOT and M-MOT can be observed.

The MOT position should be coincident with the zero point of the magnetic field, which is produced by an Anti-Helmholtz coil pair. Each coil is $6.3 \mathrm{~cm}$ in diameter, $1 \mathrm{~cm}$ in width, and is wound 75 turns $/ \mathrm{cm}$. Each coil is placed with a separation of $5.8 \mathrm{~cm}$. Finally, the current of $4.5 \mathrm{~A}$ in this configuration creates a magnetic field with a $9.1 \mathrm{G} / \mathrm{cm}$ gradient. It should be noted that when the trap coil axis is parallel with the reflected beam, the M-MOT will be efficient [10].

Fig. 5 shows twenty optical components aligned on

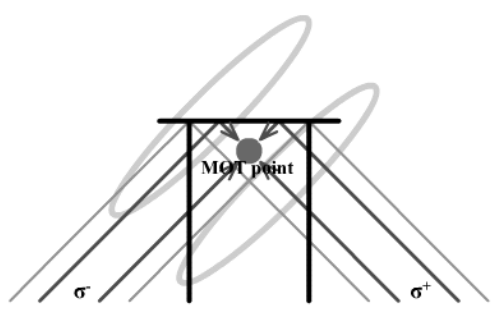

(a)

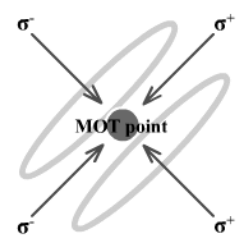

(b)
FIG. 4. (a) M-MOT beam alignment, (b) conventional MOT (six beam) beam alignment.

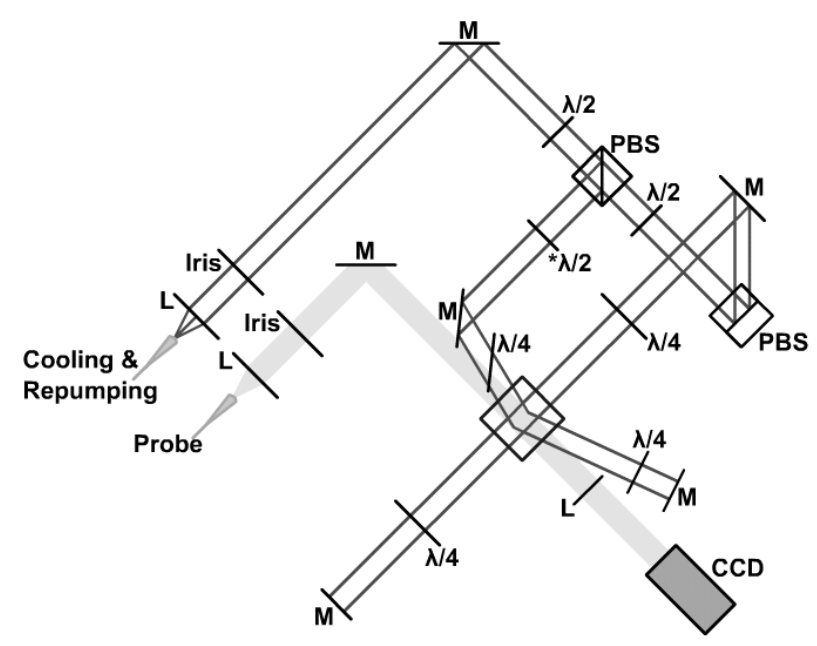

FIG. 5. Optical alignment for M-MOT.

a $50 \times 60 \mathrm{~mm}^{2}$ table for the M-MOT. A $\mathrm{f}=15 \mathrm{~mm}$ convex lens, which is anti-reflection coated for $780 \mathrm{~nm}$, is installed for beam collimation in front of the optical fiber for the cooling beam. The final beam is about $2.5 \mathrm{~cm}$ in diameter, and only $40 \%$ of the central part is used for the M-MOT. Each input beam's power is controlled by a $\lambda / 2$ wave plate and is circularly polarized by a $\lambda / 4$ wave plate. Finally, we can use $3.3 \mathrm{~mW} / \mathrm{cm}^{2}$ for cooling beam. It should be noted that $* \lambda / 2$ in Fig. 5 is used to maintain the polarization when the laser beam is directed up into the cuvette.

A $\mathrm{f}=75 \mathrm{~mm}$ convex lens is installed at the center position of a CCD camera and the trapped atoms. The CCD camera is located $300 \mathrm{~mm}$ away from the trapped atoms to take a 1:1 image of the trapped atoms.

\section{EXPERIMENTAL RESULTS}

Fig. 6 (a) is a side view of a cloud of trapped ${ }^{87} \mathrm{Rb}$. Considering the cuvette size, it is determined that the atomic cloud is located $5 \mathrm{~mm}$ beneath the mirror surface and its diameter is about $5 \mathrm{~mm}$. Fig. 6(b) is a diagonal view showing two atomic clouds. The cloud in the left circle is a real atomic cloud, and the other is an image reflected by the mirror. No cloud can be seen when a magnetic field is not applied, as is evident from the photo on the right. In order to obtain an absorption image, we automated the whole process through the Labview interface. Furthermore, the interface controlled all parameters for the Z-Magnetic Trap on an atom chip $[11,12]$.

Fig. 6(c) is an absorption image of an atomic cloud in which there are approximately $3 \times 10^{7}$ atoms. We took two images to calculate the temperature of the atoms by the time-of-flight method; however, owing to background noise and challenges induced by the vacuum, 

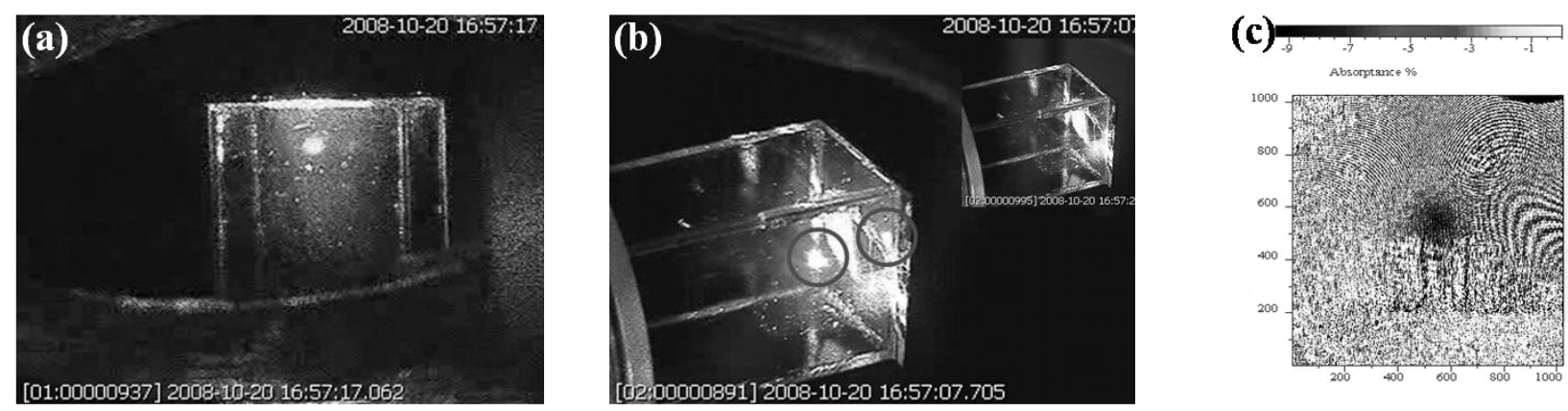

FIG. 6. Trapped ${ }^{87} \mathrm{Rb}$ atoms (a) side view (b) diagonal view, the one cloud is trapped atoms and the other is a virtual image (c) absorption image of atom cloud.

it is very difficult to obtain the exact temperature. It is thus approximately calculated as a few tens $\mathrm{mK}$. There are many $\mathrm{Rb}$ atoms located outside of the atomic clouds in the cuvette due to the use of the $\mathrm{Rb}$ getter and a great deal of light is scattered from the Pyrex surface. As a result, the absorption image has considerable background noise.

\section{CONCLUSION}

We have observed a magneto optically trapped ${ }^{87} \mathrm{Rb}$ atomic cloud having $3 \times 10^{7}$ atoms near the surface of a dielectric coated mirror at the top of a small cell, sized $20 \times 25 \times 40 \mathrm{~mm}^{3}$. Our system has many advantages including semi-permanent long lifetime under a high vacuum, polarization maintenance, realization of a small M-MOT system, easy operation of the atom chip, and short operating time. Furthermore, the interfaced system makes it possible to realize practical applied BEC system.

\section{ACKNOWLEDGMENT}

We would like to thank the KRISS and KRF for financial support.

\section{REFERENCES}

1. A. Einstein, "Strahlungs-emission und-absorption nach der quantentheorie," Deutsche Physikalische Gesellschaft 18, 318-323 (1916).
2. A. Einstein, "Quantentheorie des einaomigen idealen gasses,” Sitzungsber. Kgl. Preuss. Akad. Wiss. 261-267 (1924).

3. E. L. Raab, M. Prentiss, A. Cable, S. Chu, and D. E. Prichard, "Trapping of neutral sodium atoms with radiation pressure," Phys. Rev. Lett. 59, 2631-2634 (1987).

4. M. H. Anderson, J. R. Ensher, M. R. Matthews, C. E. Wieman, and E. A. Cornell, "Observation of BoseEinstein condensation in a dilute atomic vapor," Science 269, 198-201 (1995).

5. K. B. Davis, M. O. Mewes, M. R. Andrews, N. J. van Druten, D. S. Durfee, D. M. Kurn, and W. Ketterle, "Bose-Einstein condensation in a gas of sodium atoms," Phys. Rev. Lett. 75, 3969-3973 (1995).

6. W. Hänsel, P. Hommelhoff, T. W. Hänsch, and J. Reichel, "Bose-Einstein condensation on a microelectronic chip,” Nature 413, 498-501 (2001).

7. H. Ott, J. Fortagh, G. Schlotterbeck, A. Grossmann, and C. Zimmermann, "Bose-Einstein condensation in a surface microtrap,” Phys. Rev. Lett. 87, 230401-230404 (2001).

8. S. Du, "Atom-chip Bose-Einstein condensation in a portable vacuum cell," JILA Ph. D. thesis (2005).

9. P. D. Schwindt, "Magnetic traps and guides for BoseEinstein condensates on an atom chip-progress toward a coherent atom waveguide beamsplitter," JILA Ph.D. thesis (2003).

10. M. A. Clifford, G. P. T. Lancaster, R. H. Mitchell, F. Akerboom and K. Dholakia, "Realization of a mirror magneto optical trap,” J. Mod. Opt. 48, 1123-1128 (2001).

11. S. Schneider, A. Kasper, C. V. Hagen, M. Bartenstein, B. Engeser, T. Schumm, I. N. Joseph, R. Folman, L. Feenstra, and J. Schmiedmayer, "Bose-Einstein in a simple microtrap,” Phys. Rev. A 67, 023612-023615 (2003).

12. J. Reichel, "Microchip traps and BEC," Appl. Phys. B 75, 469-487 (2002). 